\title{
MicroRNA-26a inhibits cell proliferation and invasion of cervical cancer cells by targeting protein tyrosine phosphatase type IVA 1
}

\author{
JING DONG, LONG SUI, QING WANG, MINGJUN CHEN and HONG SUN \\ Department of Gynecology, Obstetrics and Gynecology Hospital, Shanghai Medical College, Fudan University, \\ Shanghai 200011, P.R. China
}

Received January 20, 2013; Accepted May 14, 2014

DOI: $10.3892 / \mathrm{mmr} .2014 .2335$

\begin{abstract}
The downregulation of microRNA-26a (miR-26a) has been reported in numerous types of cancer, but its detailed functional role in cervical cancer is not yet clear. In the present study, the expression of miR-26a in human cervical cancer was confirmed and its contribution to cervical cancer progression was investigated. The expression of miR-26a was determined by reverse transcription quantitative polymerase chain reaction in human cervical tissues and cell lines. Cell growth and invasion were detected by cell counting kit-8, colony-forming assays and transwell assays following restoration of miR-26a expression. Protein tyrosine phosphatase type IVA 1 (PRL-1) was further validated as a target of miR-26a by a functional luciferase assay and western blot analysis. In addition, the overexpression of miR-26a in tumor formation in SCID mice was investigated in vivo, and the association between miR-26a and PRL-1 was assayed by Pearson's correlation coefficient. First, it was identified that miR-26a was significantly downregulated in cervical cancer compared with the paired adjacent tissues. Forced expression of miR-26a suppressed cell proliferation and invasion in vitro and inhibited the growth of tumor xenografts in vivo. PRL-1 was determined as a novel target for miR-26a and knockdown of PRL-1 partially phenocopied the effect of miR-26a restoration. In addition, PRL-1 expression was inversely correlated with miR-26a expression in cervical cancer tissues. In conclusion, the results demonstrated the role of miR-26a in cervical cancer pathogenesis and suggest it may be used as a potential novel therapeutic strategy for cervical cancer.
\end{abstract}

\section{Introduction}

Cervical cancer is the second most prevalent type of cancer in females and the fourth leading cause of cancer-related mortality

Correspondence to: Professor Hong Sun, Department of Gynecology, Obstetrics and Gynecology Hospital, Shanghai Medical College, Fudan University, 419 Fangxie Road, Shanghai 200011, P.R. China

E-mail: sun321hong@163.com

Key words: cervical cancer, microRNA-26a, protein tyrosine phosphatase type IVA 1, cell growth, invasion in developing countries $(1,2)$. Although recent advances in the clinical implementation of numerous therapeutic strategies, overall 5-year survival rates remain $<40 \%$ and the molecular pathogenesis of cervical cancer is unclear (3). Therefore, investigating the mechanisms of tumor pathogenesis that contribute to disease progression may facilitate the development of novel effective therapies to prevent the occurrence and development of cervical cancer.

MicroRNAs (miRNAs) are short, non-coding RNAs that regulate gene expression at the posttranscriptional level by complementary pairing in the mRNA 3' untranslated region (3'UTR), which leads to mRNA degradation and/or translational repression $(4,5)$. Previous studies have suggested that miRNAs have an important role in numerous biological functions, including differentiation, proliferation, metastasis and apoptosis (6-9). Aberrant expression of miRNAs has been observed in human cervical cancer and several of these miRNAs have been proven as either oncogenes or tumor suppressors (10-13). Among them, miR-143, miR-145 miR-196b and miR-34a have been demonstrated to suppress cell growth, and miR-146a, miR-205, miR-182-5p and miR-21 to promote cell proliferation (11,14-16). Additionally, forced overexpression of miR-214 repressed cell growth, induced apoptosis and enhanced sensitivity to cisplatin by targeting Bcl2-12 in human cervical cancer cells (17). However, high miR-375 expression resulted in acquired paclitaxel resistance in cervical tissues (18).

Although previous studies have determined the biological functions of certain miRNAs, the sophisticated mechanism of miR-26a remains largely unknown in cervical cancer. The aim of the present study was to examine the expression of miR-26a in the cervical cancer tissues compared with the paired adjacent tissues. In addition, the biological functions were detected by ectopically expressing miR-26a, in order to identify its effect on the protein tyrosine phosphatase type IVA 1 (PRL-1) and on the activity of the MAPK pathway.

\section{Materials and methods}

Cell lines and clinical samples. Human cervical cancer cell lines (ME-180, CaSki, HeLa, SiHa), an immortalized cervical epithelial cell line (NC104), and a human embryonic kidney cell line (HEK293T) were cultured in Dulbecco's modified Eagle's medium (DMEM) supplemented with $10 \%$ (v/v) fetal 
bovine serum (FBS), $100 \mathrm{U} / \mathrm{ml}$ penicillin and $100 \mu \mathrm{g} / \mathrm{ml}$ streptomycin under a humidified atmosphere of $5 \% \mathrm{CO}_{2}$ at $37^{\circ} \mathrm{C}$.

Fresh paired human cervical tissues and adjacent normal tissues were collected from 64 patients who were undergoing surgery for cervical carcinoma between 24th July 2005 and 13th November 2008 in the Women's Hospital, School of Medicine, Fudan University (Shanghai, China). Informed consent was obtained from each patient. The study was approved and supervised by the Ethics Committee of Fudan University (Shanghai, China) and it was performed in compliance with the Helsinki Declaration. All tissue samples were snap-frozen in liquid nitrogen and stored at $-80^{\circ} \mathrm{C}$ until total RNA was extracted.

RNA extraction and reverse transcription quantitative polymerase chain reaction ( $R T-q P C R)$ analyses. Total RNA was obtained from tissue samples and cells using TRIzol reagent (Invitrogen Life Technologies, Carlsbad, CA, USA) according to the manufacturer's instructions. The relative level of miR-26a was determined by RT-qPCR using the miRCURY LNA $^{\mathrm{TM}}$ microRNA PCR system (Exiqon, Woburn, MA, USA). Briefly, 10 ng of total RNA was reverse-transcribed with the miRNA corresponding RT Primer (Exiqon, Woburn, MA, USA) and Transcriptor Reverse Transcriptase (Exiqon), and the cDNA was used as a template for the $\mathrm{qPCR}$ reaction using the miRNA specific LNA ${ }^{\mathrm{TM}}$ PCR primer (Exiqon) and Universal PCR primer (Exiqon). $\Delta \Delta \mathrm{Ct}$ values were normalized with the endogenous U6 small nuclear RNA.

For analysis of the expression of PRL-1 mRNA, $500 \mathrm{ng}$ of the total RNA was reverse transcribed using a Transcriptor First Strand cDNA Synthesis kit (Takara, Dalian, China) with random primers under standard conditions. The primers used were as follows: Sense, 5'-CACCATCTTCCAGG-AGCGAG-3' and antisense, 5'-TCACGCCACAGTTTCCCGGA-3' for GAPDH; and sense, 5'-AGGGACAAGCCTACCCCTC-3' and antisense, 5'-CTCATCTCCCGTCAGTTGGT-3' for PRL-1. GAPDH was used as the endogenous control. qPCR was performed in triplicate on the ABI Prism Sequence Detection system 7900HT (Applied Biosystems, Foster City, CA, USA). The gene expression was normalized to the internal controls and the fold-changes were calculated using relative quantification. All of the experiments were performed three times with three technical replicates.

Plasmid constructs and luciferase assay. To construct the miR-26a overexpression vector, a DNA fragment encoding the miR-26a pre-miRNA was amplified by PCR using the following oligonucleotide primers: Sense: 5'-GGCGAA TTCCCCACTGCTGACCCATTC-3' and antisense: 5'-TATGG ATCCCCACAAGACTCCTCGTTGC-3'. The PCR product was $\mathrm{TOPO}^{\circledR}$-cloned into the $\mathrm{pCR}^{\circledR} 4-\mathrm{TOPO}^{\circledR}$ vector (Invitrogen Life Technologies). The construct was sequenced and the pre-hsa-miR-26a fragment was sub-cloned into pcDNA4/myc-HisA to generate pcDNA4/miR-26a.

The human PRL-1 3'UTR luciferase reporter, containing putative binding sites for miR-26a, was generated by cloning the PRL-1 mRNA3'UTR sequence into the downstream of the luciferase gene of the pGL3-control vector (Promega Corporation, Madison,WI,USA) using the following primers: Sense: 5'-GGC TCTAGAGGGCCTACAGGAGGGGTTA-3' and antisense: 5'-GGCTCTAGATGTGATTAAAGTAAAATGCA
ATTCA-3'. The plasmids was termed PRL-1-UTR-WT and site-directed mutagenesis of the miR-26a target site in the PRL-1 3'UTR was performed using the Quick-change mutagenesis kit (Stratagene, La Jolla, CA, USA) to generate the PRL-1-UTR-MUT plasmids. Correct vector construction was verified by direct sequencing. For the mutated construct, the miR-26a target site UACUUGAC was substituted with a UAGAACAC fragment.

To silence the PRL-1 expression, lentivirus vector shPRL-1 was constructed to establish PRL-1 silencing as described previously (19) using the following primer sequences: Sense: 5'-CCGGTTCTTGCTGTCAGCATATAAACTCGA GTTTATATGCTGACAGCAAGAATTTTTG-3' and antisense: 5'-AATTCAAAAATTCTTGCTGTCAGCATATAAA CTCGAGTTTATATGCTGACAGCAAGAA-3'.

A dual luciferase assay was conducted by co-transfecting HEK293T cells with $20 \mathrm{ng}$ pcDNA4/miR-26a or empty lentiviral vector, along with $100 \mathrm{ng}$ of firefly luciferase reporter comprising wild type or mutant 3'UTR of PRL-1 gene and $10 \mathrm{ng}$ pRL-TK vector, using Lipofectamine ${ }^{\mathrm{TM}} 2000$ (Invitrogen Life Technologies) per well, according to the manufacturer's instructions in a 24-well plate format. The cells were harvested $48 \mathrm{~h}$ following transfection for luciferase assay using a luciferase assay kit (Promega Corporation) and Renilla luciferase activity was used for transfection variation normalization according to the manufacturer's instructions. Each experiment was repeated three times.

Cell proliferation assays. The cells were seeded at a density of 2,000 cells per well in a 96-well plate containing $100 \mu \mathrm{l}$ DMEM culture media with $10 \%$ FBS. A total of $10 \mu \mathrm{l}$ cell counting kit (CCK)-8 solution was added to each well and the cells were incubated at $37^{\circ} \mathrm{C}$ for $2 \mathrm{~h}$. The absorbance values were measured at $450 \mathrm{~nm}$ every $24 \mathrm{~h}$ following the manufacturer's instructions. Triplicate wells were measured in each treatment group.

Matrigel invasion assay. A total of $4 \times 10^{4}$ cells in $100 \mu \mathrm{l}$ serum free media were seeded into the upper chamber coated with $150 \mu \mathrm{g}$ of Matrigel (BD Biosciences, Bedford, MD, USA), and NIH 3T3 fibroblast conditioned medium was added to the lower chamber. After the cells were incubated for $36 \mathrm{~h}$, noninvasive cells were removed from the upper surface of the membrane with a cotton swab, and the invaded cells on the lower membrane surface were fixed and stained with hematoxylin for $30 \mathrm{~min}$. Following drying, the invasive cells were captured using a microscope in five random fields using a DP controller (Olympus, Tokyo, Japan).

Colony formation assay. The cells were placed in 6-well plates at a density of 500 cells per well in normal culture medium as stated above and incubated for two weeks, the medium was replaced every four days. Next the cells were washed twice with PBS, fixed with $4 \%$ polyoxymethylene and stained with $1 \%$ crystal violet for $30 \mathrm{~min}$. The number of colonies was counted and a single clone contained $>50$ cells. Each assay was performed in triplicate.

Western blot analysis. The cells were washed twice with PBS and then lysed using RIPA buffer supplemented with protease inhibitor cocktail (Roche, Basel, Switzerland) and sonicated 
(Shengyan, Shanghai, China) with one $10 \mathrm{sec}$ burst. Whole cell extracts containing equal quantities of proteins $(50 \mu \mathrm{g})$ were separated by $10 \%$ SDS-polyacrylamide gel electrophoresis (SDS-PAGE), transferred to $0.45-\mu \mathrm{m}$ polyvinylidene difluoride membrane sheets (Millipore, Billerica, MA, USA). The membrane was blocked with 5\% skimmed milk at room temperature for $1 \mathrm{~h}$ and probed with the following antibodies: Anti-PRL-1 antibody (ab3523, Abcam, Cambridge, UK) and anti-GAPDH antibody (sc-25778; Santa Cruz Biotechnology, Inc., Santa Cruz, CA, USA). Following an overnight incubation, the membrane was washed and incubated with the appropriate goat anti-rabbit horseradish peroxidase-conjugated secondary antibody (Kangcheng, Shanghai, China) at room temperature for $1 \mathrm{~h}$. The protein bands were subjected to a chemiluminescence detection assay (chemiluminescence kit; Tiangen, Beijing, China).

Nude mouse tumor xenograft model. The present study was conducted in accordance with the Care and Use of Laboratory Animals of the National Institutes of Health. All of the experimental procedures were approved by the Committee on the Care and Use of Laboratory Animals of the Fudan University. Female nude mice (age, 3-5 weeks) were purchased from Shanghai SLAC Laboratory Animal Co. Ltd. (Shanghai, China). The mice were housed under humidity- and temperature-controlled conditions with a $12 \mathrm{~h}$ light/dark cycle, and were fed a normal diet. Xenografts were established by subcutaneous injection of $5 \times 10^{6}$ Hela cells with vector control or overexpression miR-26a per mouse in the right flank area in a volume of $200 \mu \mathrm{l} \mathrm{FBS-free} \mathrm{DMEM} \mathrm{medium.} \mathrm{The} \mathrm{animals}$ were sacrificed by joint dislocation following six weeks and the tumor volume was measured using a caliper every seven days, using the following formula: Volume $=(\text { width })^{2} \times$ length $/ 2$. Four mice were used in each group and the experiment was repeated three times independently.

Statistical analysis. The experimental data were demonstrated as the mean \pm standard deviation for each group. Student's t-test (two-tailed) was performed to analyze the data using GraphPad Prism software (La Jolla, CA, USA). $\mathrm{P}<0.05$ were considered to indicate a statistically significant difference.

\section{Results}

miR-26a is significantly downregulated in primary cervical cancer tissues and cell lines. In order to assess the roles of miR-26a in human cervical cancer development, the miR-26a expression levels in cervical cancer tissues $(n=64)$ and paired adjacent normal tissues were compared by RT-qPCR. It was identified that miR-26a expression was markedly reduced in tumor tissues as compared with that in adjacent normal tissues (Fig. 1A). Furthermore, the expression level of miR-26a in several human cervical cancer cell lines and the NC104 human normal immortalized cervical epithelial cell line was detected. As expected, the expression of miR-26a was decreased in all of the cancer cell lines compared with that in the normal cells (Fig. 1B). These results indicated that miR-26a was significantly downregulated in clinical human cervical cancer tissues and cells, and therefore, may be involved in human cervical cancer development.
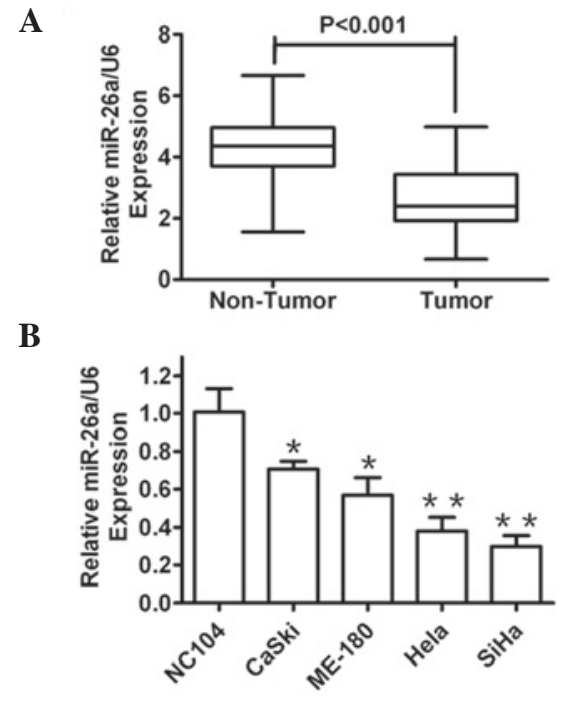

Figure 1. Decreased expression of miR-26a in clinical specimens of human cervical cancer and cancer cells. (A) The expression of miR-26a was downregulated in cervical cancer tissues compared with the adjacent normal tissues (n=64). (B) The expression levels of miR-26a were notably lower in cervical cancer cells, particularly in Hela and SiHa cells. The expression of miR-26a was normalized to U6 RNA expression in each sample. Data are expressed as the mean \pm standard deviation. ${ }^{*} \mathrm{P}<0.05$ and ${ }^{* *} \mathrm{P}<0.01$, compared with control vector. miR-26a, microRNA-26a.

miR-26a overexpression suppresses proliferation and invasion of cervical cancer cells. To elucidate the potential mechanisms underlying the effects of miR-26a in cervical cancer cells, miR-26a was restored in the Hela and $\mathrm{SiHa}$ cell lines by a lentiviral vector. miR-26a transduction significantly increased the miR-26a expression in Hela and $\mathrm{SiHa}$ cells compared with the vector cells, as demonstrated by RT-qPCR analysis (Fig. 2A), reflecting efficient overexpression of miR-26a in these cervical cancer cells. Next, several functional analyses were performed. A CCK-8 assay demonstrated that forced expression of miR-26a significantly reduced cell proliferation in the two cervical cancer cell lines (Fig. 2B and C). To further evaluate the proliferation ability, the effects of the restoration of miR-26a on colony formation were examined. As demonstrated in Fig. 2D, there were notably fewer and smaller colonies of the cells overexpressing miR-26a compared with the vector transductions.

Invasion is the key element of tumor metastasis, thus it was investigated whether reintroducing miR-26a affects cell invasion by utilizing transwell migration assays. As exhibited in Fig. 2E, miR-26a significantly reduced the number of Hela and $\mathrm{SiHa}$ cells invading through the Matrigel basement membrane. Together, these observations suggested that the loss of miR-26a in cervical cancer may, at least partially, contribute to tumor growth and invasive capacity.

miR-26a inhibits tumor growth in an allograft murine model. Considering the aforementioned results that demonstrated that forced expression of miR-26a inhibited cell growth in cervical cancer cells in vitro, an in vivo model was used to examine the effect of miR-26a restoration on tumor growth. It was identified that miR-26a restoration markedly inhibited tumor formation and reduced the tumor size and weight after five weeks compared with the vector control (Fig. 3A and B). 
A

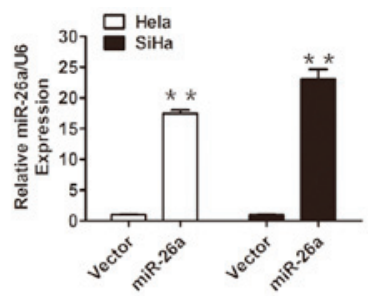

D

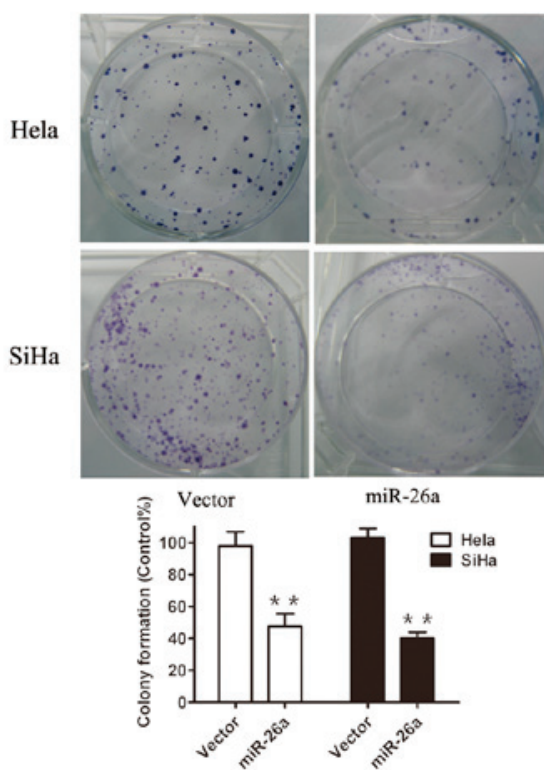

B

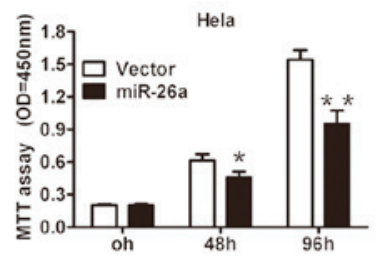

C

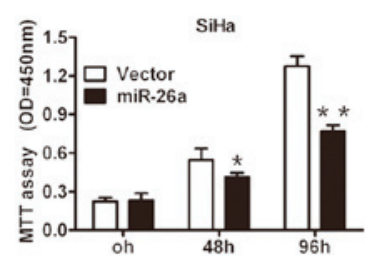

$\mathbf{E}$

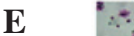

Hela

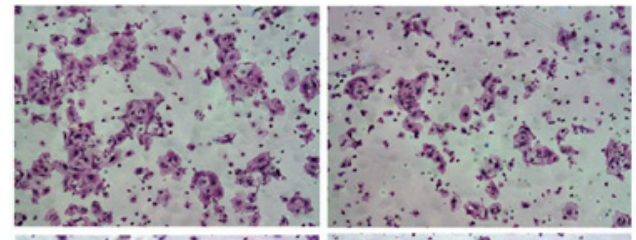

$\mathrm{SiHa}$

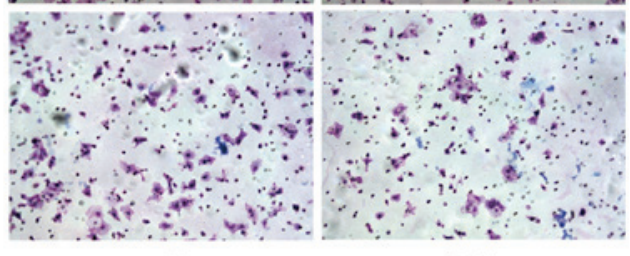

Vector

miR-26a

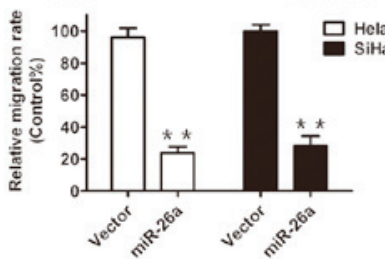

Figure 2. miR-26a suppressed cell proliferation. (A) Overexpression of miR-26a was confirmed by quantitative polymerase chain reaction. The cell proliferation assay demonstrated that ectopic expression of miR-26a significantly reduced the growth rates of (B) Hela and (C) SiHa cells. (D) Enforced expression of miR-26a markedly decreased the number and size of Hela and SiHa cells. Quantitative analysis of colony numbers is demonstrated, with values expressed as the mean \pm standard deviation. (E) Representative images exhibit invasive cells following infection with miR-26a or vector. The cells were counted and analyzed with a histogram. All of the experiments were performed at least three times independently. ${ }^{*} \mathrm{P}<0.05$ and ${ }^{* * *} \mathrm{P}<0.01$, compared with control vector. miR-26a, microRNA-26a.

A

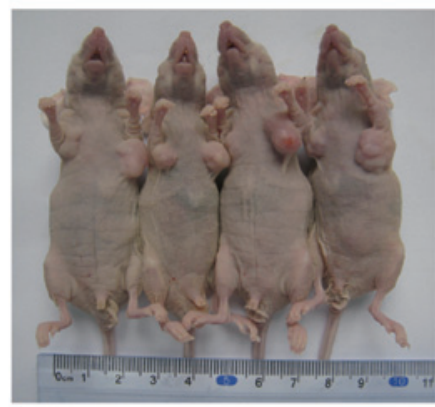

B

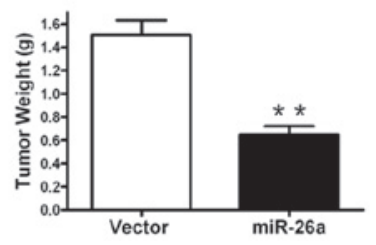

C

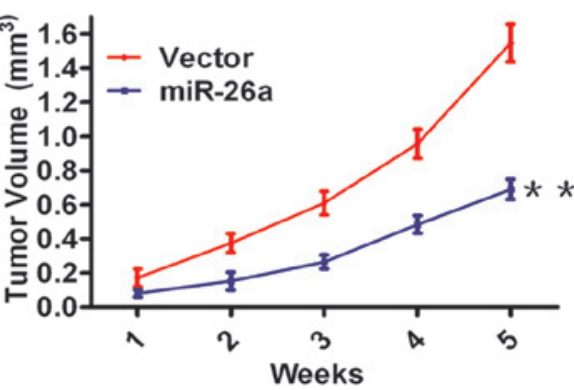

Figure 3. miR-26a inhibited tumor growth in vivo. (A) miR-26a or vector infected Hela cells were injected subcutaneously into the flank of nude mice and the tumor size and volume were evaluated at six weeks. (B and C) The weight and growth curve of tumor xenografts were calculated following miR-26a overexpression. ${ }^{* *} \mathrm{P}<0.01$, compared with the control vector. miR-26a, microRNA-26a.

These data indicate that overexpression of miR-26a reduces tumor growth in Hela cells in vivo.

miR-26a directly targets PRL-1 in cervical cancer cells. To examine the mechanisms through which miR-26a regulated cervical cancer cell growth, two publicly available algorithms were used to facilitate predicting the miR-143 targets. A bioinformatic prediction (Targetscan and Pictar) for putative targets of miR-26a was used and it was identified that PRL-1 was a potential target of miR-34a. Increased expression of PRL-1 has been reported and implicated in tumor progression and angiogenesis in human lung cancer and colon cancer (20). The present study focused on the possible regulation of PRL-1 by miR-26a. Sequence analyses revealed that the 3'-UTR of PRL-1 mRNA was partially complementary to miR-26a (Fig. 4A). To experimentally validate the direct miR-26a-PRL-1 interaction, PRL-1-UTR-WT and PRL-1-UTR-MUT plasmids were constructed. Upregulation of miR-26a led to a significant decrease in luciferase activity when the reporter contained a wild-type sequence, but no change in the luciferase activity of the mutant sequence (Fig. 4B). Furthermore, the endogenous expression of PRL-1 in Hela and SiHa cells upon miR-26a forced expression was examined, and it was identified that the endogenous PRL-1 protein level was markedly reduced by 
A

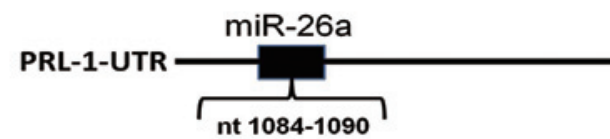

MUT 5' -AACUUAAUAUUUGGAUAGAACAC-3'

WT 5'-AACUUAAUAUUUGGAUACUUGAC-3'

| || || ||

Has-miR-26a 3'-UCGGAUAGGACCUAAUGAACUU-5'

B

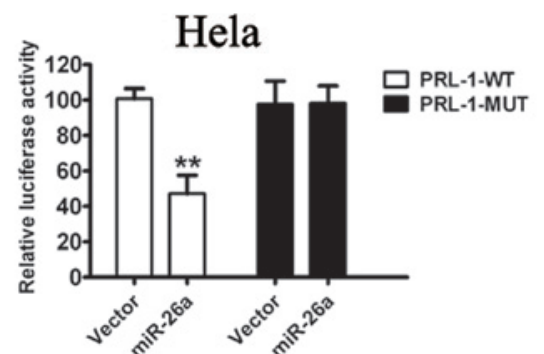

C

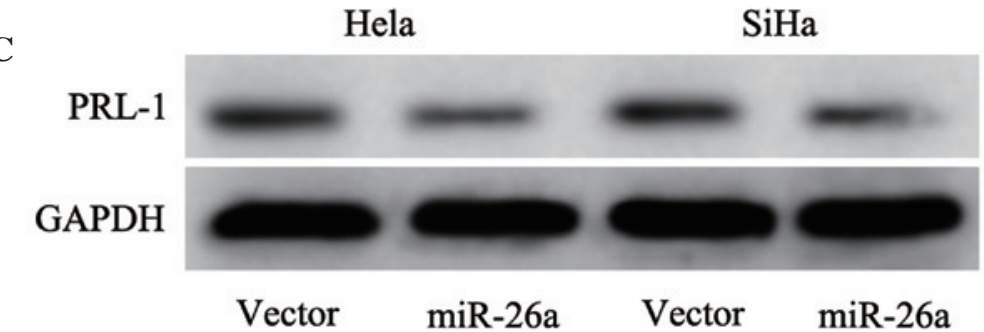

$\mathrm{SiHa}$

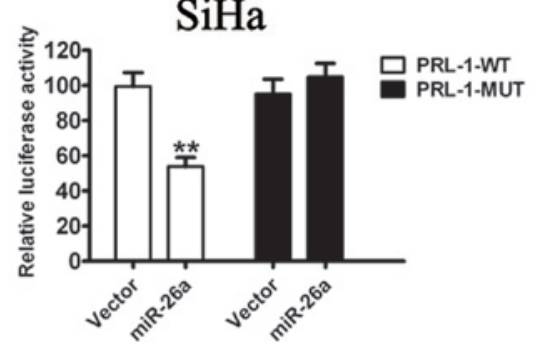

$\mathrm{SiHa}$

Figure 4. PRL-1 is a target of miR-26a. (A) Computational algorithm demonstrating the seed region of miR-34a in the PRL-1 3'UTR. (B) The dual luciferase reporter assay of HEK-293 T cells co-transfected with PRL-1-UTR-WT or PRL-1-UTR-MUT and miR-26a or vector, and the pRL-TK were normalized to the internal firefly luciferase transfection control. (C) The protein expression of PRL-1 was detected by western blot analysis in Hela and SiHa cells. Data are presented as the mean \pm standard deviation of three independent experiments. " $\mathrm{P}<0.05$ and ${ }^{* *} \mathrm{P}<0.01$, compared with control vector. PRL-1, protein tyrosine phosphatase type IVA 1; miR-26a, microRNA-26a; 3'UTR, 3' untranslated region.

miR-26a (Fig. 4C). These results demonstrated a direct interaction between miR-26a and PRL-1 in cervical cancer cell lines.

Knockdown of PRL-1 expression phenocopies the effects of $\mathrm{miR}-26$ a restoration in cervical cancer cells. To evaluate whether PRL-1 is potentially involved in miR-26a-regulated cell proliferation and invasiveness, shPRL-1 efficiently knocked down endogenous PRL-1 expression (Fig. 5). Compared with the negative control, silencing PRL-1 markedly suppressed cell proliferation (Fig. 5A and B), colony formation (Fig. 5C) and invasive ability (Fig. 5D) of Hela and SiHa cells. As PRL-1, a direct target of miR-26a, has an important role in extracellular signal regulated kinases (ERK)1/2 activation (21), the expression of ERK1/2 and the downstream effectors following PRL-1 silencing were detected. As expected, PRL-1 knockdown reduced ERK1/2 and RhoA activation in the cervical cancer cells (Fig. 5E). To further confirm that PRL-1 is a target gene protein of miR-26a, it was investigated whether miR-26a affected the expression of ERK1/2 and RhoA through suppression of PRL-1 expression. Consistent with the results of PRL-1 silencing, miR-26a overexpression significantly decreased the expression of ERK1/2 and RhoA (Fig. 5F). These results provide evidence suggesting that PRL-1 is involved in miR-26a-regulated cervical cancer cell growth and invasiveness.

Upregulation of PRL-1 expression is inversely correlated with miR-26a in primary cervical cancer tissues. To further deter- mine the correlation between miR-26a and PRL-1, the mRNA level of PRL-1 in primary human cervical cancer tissues was detected and compared with that in the normal adjacent tissue in the same patient cohort that was used for measuring the miR-26a levels. The expression of PRL-1 was markedly higher in the tumor tissue compared with the adjacent normal tissue (Fig. 6A). Notably, there was an inverse correlation between the expression level of miR-26a and PRL-1 in the cervical cancer tissues, as determined by Pearson's correlation coefficient (Fig. 6B). Collectively, these data supported the evidence for a reciprocal correlation between the levels of miR-26a and PRL-1 in human cervical cancer.

\section{Discussion}

Previous studies have reported that aberrant expression of miR-26a is a common feature of a wide spectrum of human malignancies and is associated with poor prognosis in certain cancer types, including breast cancer (22), nasopharyngeal carcinoma (23), hepatocellular carcinoma (24), breast cancer (25), gliomagenesis (26), cholangiocarcinoma (27) and lung cancer (28). miR-26a may act as a tumor suppressor or oncogene in different tumor types, which may owe to unique genetic backgrounds and/or it not being conserved in certain types of cancer cells. miR-26a has been reported to be reduced in human cervical cancer tissues, compared with normal cervical tissues or adjacent benign cervical tissues. In the 
A

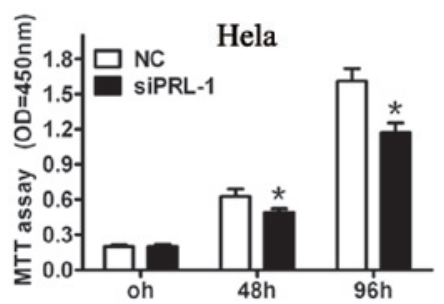

C

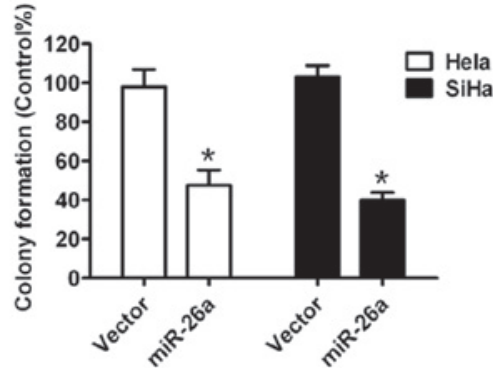

$\mathbf{E}$

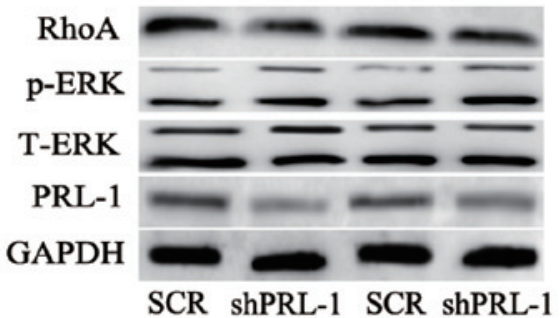

B

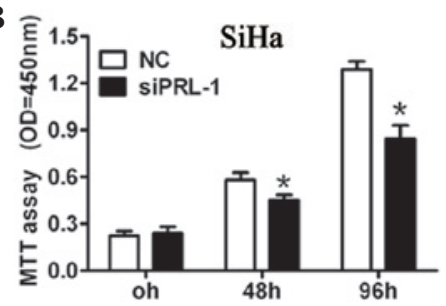

D

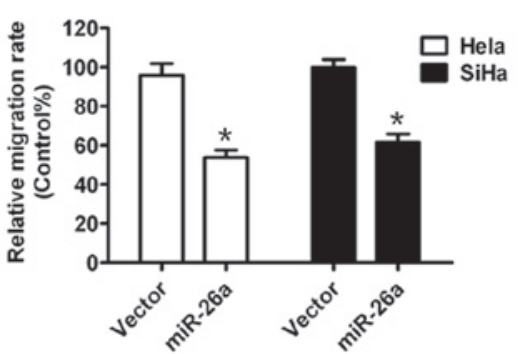

F

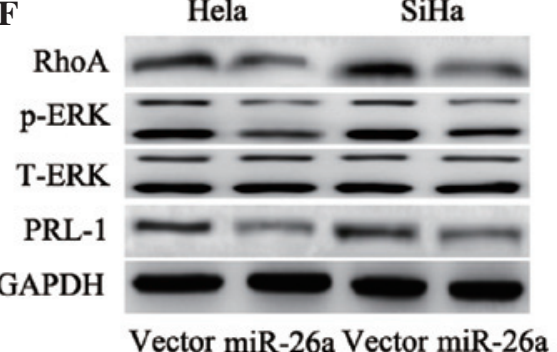

Figure 5. Knockdown of PRL-1 suppresses growth, migration and invasion of cervical cancer cells. The proliferative capacity of (A) Hela and (B) SiHa cells following knockdown of PRL-1 was significantly lower than that of cells infected with SCR. (C) Colony formation analysis of cell growth in Hela/shPRL-1 (or Hela/SCR) or SiHa/shPRL-1 (or SiHa/ SCR) cells. (D) Cell migration ability of Hela and SiHa cells infected with shPRL-1 or SCR was assessed by transwell migration analysis. (E) Western blot analysis of PRL-1, p-ERK and RhoA following PRL-1 silencing. (F) Western blot analysis demonstrating reduced PRL-1, p-ERK and RhoA levels following miR-26a overexpression. ${ }^{*} \mathrm{P}<0.05$ and ${ }^{* *} \mathrm{P}<0.01$, compared with control vector. miR-26a, microR $\mathrm{NA}-26 \mathrm{a}$; PRL-1, protein tyrosine phosphatase type IVA 1; SCR, negative control; ERk, extracellular signal-regulated kinases.

A

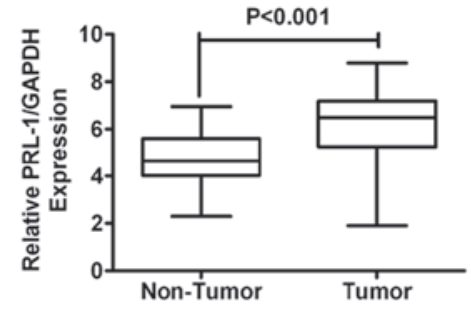

B

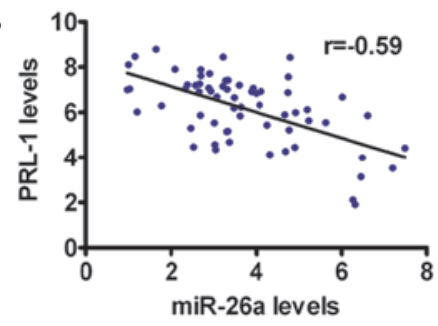

Figure 6. Expression of PRL-1 was upregulated in cervical cancer tissues and correlated with miR-26a. (A) The expression of PRL-1 was examined by quantitative polymerase chain reaction and normalized to GADPH expression. (B) Negative correlation between miR-26a and the mRNA level of PRL-1 ( $\mathrm{n}=64$ ). Statistical analysis was performed using Pearson's correlation coefficient assay using GraphPad Prism software. miR-26a, microRNA-26a; PRL-1, protein tyrosine phosphatase type IVA 1.

present study, the action of miR-26a in cervical cancer lines was examined.

In the present study, miR-26a was identified as a tumor suppressor in cervical cancer through directly targeting PRL-1, which is known to upregulate ERK1/2 and RhoA pathways (21). First the expression of miR-26a was determined and it was identified that miR-26a was significantly lower in cervical cancer tissues than in the corresponding adjacent tissues. As is consistent with the results in the tissue samples, the miR-26a expression demonstrated a marked attenuation in the cervical cancer cell lines, particularly in Hela and SiHa cells. Furthermore, forced expression of miR-26a inhibited the proliferation and invasion of cervical cancer cells in vitro. These results are consistent with previous studies that have demonstrated that miR-26a was downregulated in several cancer tissues and was identified as a tumor suppressor (12,23-25). Systemic administration of miR-26a AAV by tail vein injection into mice led to tumor suppression without any toxic effects when assessed three weeks later (29). The present results indicated that miR-26a may be an attractive therapeutic agent for cervical cancer treatment. 
With use of bioinformatics prediction and sequence analyses, PRL-1 was considered to be direct target of miR-26a in cervical cancer. In luciferase reporter assays, miR-26a was able to suppress luciferase activity in the PRL-1 WT but had no effect in the mutant construct. Exogenous overexpression of miR-26a confirmed the decrease in PRL-1 expression. Upregulation of PRL-1 expression has been reported in several distinct cancer types, including hepatocellular carcinoma (30), colorectal carcinoma (20) and lung cancer (31). However, the expression and roles of PRL-1 in cervical cancer remain unclear. Higher expression of PRL-1 in cervical cancer tissue relative to the normal adjacent cervical tissue was observed, suggesting that upregulation of PRL-1 expression in cervical cancer may be implicated in processes that are essential for tumor cell growth and metastasis due to their ability to affect cell migration. Silencing PRL-1, by downregulating its expression, decreased the cell proliferation and invasion with decreased expression of p-ERK and inactivation of RhoA. Consistent with PRL-1 knockdown, miR-26a overexpression also reduced the expression of p-ERK and inactivation of RhoA. Additionally, miR-26a expression was inversely correlated with the PRL-1 mRNA expression in cervical cancer tissues.

In the present study, it was identified that miR-26a was reduced in cervical cancer tissues and cervical cancer cells. Furthermore, the overexpression of miR-26a suppressed cervical cancer cell growth in vitro and in vivo. Although the different biological functions of miR-26a have been previously reported, its function in cervical cancer remained unclear. To the best of our knowledge, the present study is the first to demonstrate the role of miR-26a in cervical cancer. Together with previous studies, the present results confirm that miR-26a is a tumor suppressor that has a key role in the initiation and progression of human cervical cancer by affecting the expression of PRL-1. Further investigation of the association between miR-26a with the clinicopathological parameters of cervical cancer is required to address the clinical applications of these data. The determination of the functions of miR-26a may aid the development of future therapeutics for cervical cancer.

\section{Acknowledgements}

This study was supported by the National Natural Science Foundation of China (grant no. 81272877) and the Cancer Foundation of China. The funders had no role in the study design, data collection, analysis, decision to publish or preparation of the manuscript.

\section{References}

1. Jemal A, Bray F, Center MM, et al: Global cancer statistics. CA Cancer J Clin 61: 69-90, 2011.

2. Parkin DM: Global cancer statistics in the year 2000. Lancet Oncol 2: 533-543, 2001.

3. Saslow D, Solomon D, Lawson HW, et al: American Cancer Society, American Society for Colposcopy and Cervical Pathology, and American Society for Clinical Pathology screening guidelines for the prevention and early detection of cervical cancer. Am J Clin Pathol 137: 516-542, 2012.

4. Farh KK, Grimson A, Jan C, et al: The widespread impact of mammalian MicroRNAs on mRNA repression and evolution. Science 310: 1817-1821, 2005.

5. Bartel DP: MicroRNAs: genomics, biogenesis, mechanism, and function. Cell 116: 281-297, 2004
6. MarziMJ,PuggioniEM,Dall'OlioV,etal:Differentiation-associated microRNAs antagonize the Rb-E2F pathway to restrict proliferation. J Cell Biol 199: 77-95, 2012.

7. Kane NM, Howard L, Descamps B, et al: Role of microRNAs 99b, $181 \mathrm{a}$, and $181 \mathrm{~b}$ in the differentiation of human embryonic stem cells to vascular endothelial cells. Stem Cells 30: 643-654, 2012.

8. Zhu XC, Dong QZ,Zhang XF, et al: microRNA-29a suppresses cell proliferation by targeting SPARC in hepatocellular carcinoma. Int J Mol Med 30: 1321-1326, 2012.

9. Cheng AM, Byrom MW, Shelton J and Ford LP: Antisense inhibition of human miRNAs and indications for an involvement of miRNA in cell growth and apoptosis. Nucleic Acids Res 33: 1290-1297, 2005

10. Lee JW, Choi CH, Choi JJ, et al: Altered MicroRNA expression in cervical carcinomas. Clin Cancer Res 14: 2535-2542, 2008.

11. Wang X, Tang S, Le SY, et al: Aberrant expression of oncogenic and tumor-suppressive microRNAs in cervical cancer is required for cancer cell growth. PLoS One 3: e2557, 2008.

12. Pereira PM, Marques JP, Soares AR, Carreto L and Santos MA: MicroRNA expression variability in human cervical tissues. PLoS One 5: e11780, 2010.

13. Gocze K, Gombos K, Juhasz K, et al: Unique microRNA expression profiles in cervical cancer. Anticancer Res 33: 2561-2567, 2013.

14. Hirata H, Ueno K, Shahryari V, et al: Oncogenic miRNA-182-5p targets Smad4 and RECK in human bladder cancer. PLoS One 7: e51056, 2012

15. Xie H, Zhao Y, Caramuta S, Larsson C and Lui WO: miR-205 expression promotes cell proliferation and migration of human cervical cancer cells. PLoS One 7: e46990, 2012.

16. Wang X, Wang HK, McCoy JP, et al: Oncogenic HPV infection interrupts the expression of tumor-suppressive miR-34a through viral oncoprotein E6. RNA 15: 637-647, 2009.

17. Wang F, Liu M, Li X and Tang H: MiR-214 reduces cell survival and enhances cisplatin-induced cytotoxicity via down-regulation of Bcl212 in cervical cancer cells. Febs Lett 587: 488-495, 2013.

18. Shen Y, Wang P, Li Y, et al: miR-375 is upregulated in acquired paclitaxel resistance in cervical cancer. Br J Cancer 109: 92-99, 2013.

19. Dong J, Cheng M and Sun H: Function of inducible nitric oxide synthase in the regulation of cervical cancer cell proliferation and the expression of vascular endothelial growth factor. Mol Med Rep 9: 583-589, 2014.

20. Fiordalisi JJ, Keller PJ and Cox AD: PRL tyrosine phosphatases regulate rho family GTPases to promote invasion and motility. Cancer Res 66: 3153-3161, 2006.

21. Bai Y, Luo Y, Liu S, et al: PRL-1 protein promotes ERK1/2 and RhoA protein activation through a non-canonical interaction with the Src homology 3 domain of p115 Rho GTPase-activating protein. J Biol Chem 286: 42316-42324, 2011.

22. Chen HC, Chen GH, Chen YH, et al: MicroRNA deregulation and pathway alterations in nasopharyngeal carcinoma. Br J Cancer 100: 1002-1011, 2009.

23. Lu J, He ML, Wang L, et al: MiR-26a inhibits cell growth and tumorigenesis of nasopharyngeal carcinoma through repression of EZH2. Cancer Res 71: 225-233, 2011

24. Yang X, Liang L, Zhang XF, et al: MicroRNA-26a suppresses tumor growth and metastasis of human hepatocellular carcinoma by targeting interleukin-6-Stat3 pathway. Hepatology 58: 158-170, 2013.

25. Zhang B, Liu XX, He JR, et al: Pathologically decreased miR-26a antagonizes apoptosis and facilitates carcinogenesis by targeting MTDH and EZH2 in breast cancer. Carcinogenesis 32: 2-9, 2011.

26. Huse JT,Brennan C,HambardzumyanD, etal:ThePTEN-regulating microRNA miR-26a is amplified in high-grade glioma and facilitates gliomagenesis in vivo. Genes Dev 23: 1327-1337, 2009.

27. Zhang J, Han C and Wu T: MicroRNA-26a promotes cholangiocarcinoma growth by activating beta-catenin. Gastroenterology 143 : 246-256, 2012.

28. Liu B, Wu X, Liu B, et al: MiR-26a enhances metastasis potential of lung cancer cells via AKT pathway by targeting PTEN. Biochim Biophys Acta 1822: 1692-1704, 2012.

29. Kota J, Chivukula RR, O'Donnell KA, et al: Therapeutic microRNA delivery suppresses tumorigenesis in a murine liver cancer model. Cell 137: 1005-1017, 2009.

30. Lu JW, Chang JG, Yeh KT, et al: Increased expression of PRL-1 protein correlates with shortened patient survival in human hepatocellular carcinoma. Clin Transl Oncol 14: 287-293, 2012.

31. Achiwa $\mathrm{H}$ and Lazo JS: PRL-1 tyrosine phosphatase regulates c-Src levels, adherence, and invasion in human lung cancer cells. Cancer Res 67: 643-650, 2007. 